\title{
Semiclassical Quantization of a System with Mixed Regular/Chaotic Dynamics
}

\author{
J. L. Schoendorff and H. J. Korsch \\ Fachbereich Physik, Universität Kaiserslautern, D-67653 Kaiserslautern, Germany
}

\begin{abstract}
N. Moiseyev
Department of Chemistry and Minerva Center for Non Linear Physics of Complex Systems, Technion-Israel Institute of Technology, Haifa 32000, Israel
\end{abstract}

(March 17, 1998)

\begin{abstract}
The quasienergy spectrum of a periodically driven quantum system is constructed from classical dynamics by means of the semiclassical initial value representation using coherent states. For the first time, this method is applied to explicitly time dependent systems. For an anharmonic oscillator system with mixed chaotic and regular classical dynamics, the entire quantum spectrum (both regular and chaotic states) is reproduced semiclassically with surprising accuracy. In particular, the method is capable to account for the very small tunneling splittings.
\end{abstract}

PACS number(s): 05.45.+b, 03.65.Sq 
The classical-quantum correspondence for typical systems with mixed regular/chaotic classical dynamics is still a challenging problem of current research. We have demonstrated a surprisingly high degree of such a correspondence for time-periodically driven systems [1-4]. Here we consider the problem of semiclassical quantization of quasienergy states for such systems.

A semiclassical theory of quasienergy states has been presented recently $[5,6]$ for integrable systems based on classical invariant tori. The method can be extended to (weakly) chaotic systems to quantize semiclassically the regular quantum states still supported by invariant tori. It is, however, not possible to treat chaotic states by such a technique. or systems with mixed dynamics.

In recent studies, quantum systems have been successfully treated semiclassically by the semiclassical initial value representation [7] using a frozen Gaussian approximation (FGA) [8]. The papers by Herman and Kluk [9,10], by Miller and coworkers [11] as well as studies by Grossman [12], Garashchuk and Tannor [13], Walton and Manolopoulos [14] and Kay [15] demonstrate the wide applicability of this method. Additional progress has been made by combination with the cellular dynamics algorithm of Heller $[16,17,20]$.

An advantage of the FGA is the absence of the difficulties related to root searching and uniformization in the somewhat more rigorous semiclassical approach, which aims at the construction of all elements of the analysis in the semiclassical limit. The FGA method also seems to work for systems which are classically chaotic [14]. Previous application have shown the applicability of the method for systems depending not explicitly on time [11-17] (see [18] for a recent review; a different, however related approach based on classically propagated Gaussians has been suggested by Pattanayak and Schieve [19]).

Here we extend such a hybrid semiclassical method to time dependent systems, more specifically we construct the quasienergy (Floquet) states of a periodically driven quantum system, which is typical in the sense that it shows mixed regular and chaotic motion classically. The method is entirely different from the 'rigorous' semiclassical periodic orbit quantization [21] and avoids the problems related to the proliferation of the long periodic 
orbits. A different approach avoiding long periodic orbits has been recently used to construct the spectrum of a kicked top semiclassically [22]. A related semiclassical description of the quasienergy states of the kicked rotor has been developed by Chang and Perez [23]. Here we report a general semiclassical technique which is easily applicable for continuously driven systems.

As an example, we consider a simplified version of an anharmonic oscillator coupled to an external driving field

$$
\begin{aligned}
H= & \omega \hat{a}^{\dagger} \hat{a}+\chi \hat{a}^{\dagger 2} \hat{a}^{2}+g_{1}\left(\hat{a} \mathrm{e}^{\mathrm{i} \Omega t}+\hat{a}^{\dagger} \mathrm{e}^{-\mathrm{i} \Omega t}\right) \\
& +g_{2}\left(\hat{a}^{2} \mathrm{e}^{\mathrm{i} \Omega t}+\hat{a}^{\dagger 2} \mathrm{e}^{-\mathrm{i} \Omega t}\right) .
\end{aligned}
$$

Similar Hamiltonians occur frequently in quantum optics, e.g. to model a nonlinear medium in a laser resonator driven by external lasers. The creation and annihilation operators are related to the position, $\hat{q}$, and momentum, $\hat{p}$, operators by

$$
\hat{a}=(\hat{q}+\mathrm{i} \hat{p}) / \sqrt{2}, \quad \hat{a}^{\dagger}=(\hat{q}-\mathrm{i} \hat{p}) / \sqrt{2} .
$$

The Hamiltonian (1) describes the mixing of two primary resonances zones, a 1:1 and a 1:2 resonance, as can be seen by transformation to a rotating frame.

As the coupling constants $g_{1,2}$ are increased, the two resonance zones overlap creating higher order resonances until finally an extended part of the phase space begins to show mixed regular/chaotic motion (compare Fig. 1). Since the Hamiltonian is periodic with period $T=2 \pi / \Omega$, the dynamics can be understood entirely in terms of Floquet states $\left|\psi_{\alpha}(t)\right\rangle$ (see, e.g. $[5,6,24]$ ), which are eigenvectors of the Floquet Hamiltonian

$$
H_{F}\left|\psi_{\alpha}(t)\right\rangle \equiv\left(-\mathrm{i} \hbar \partial_{t}+H(t)\right)\left|\psi_{\alpha}(t)\right\rangle=\epsilon_{\alpha}\left|\psi_{\alpha}(t)\right\rangle
$$

which are $T$-periodic and live in the extended Hilbert space $\left|\psi_{\alpha}(t)\right\rangle \in \mathcal{H}_{T} \otimes \mathcal{H}_{x}$. Note that the quasienergies $\epsilon_{\alpha}$ are defined only modulo $\hbar \Omega$ and that $\alpha$ numbers the states with respect to a specified ordering (see below).

As eigenvectors of time-independent Hamiltonians are commonly accepted to correspond to certain classical trajectories so do the Floquet states $\left|\psi_{\alpha}(t)\right\rangle$. Thus a Floquet eigenstate 
$\left|\psi_{\alpha}(0)\right\rangle$ considered at time $t=0$ may be associated with a Poincaré section constructed by stroboscopically sampling the value of a certain trajectory $q(t), p(t)$ at integer multiples of the period $T$ (see Fig. 1). For the numerical calculation, we have considered the symmetrically ordered version of the Hamiltonian (1) and furthermore taken $\omega$ to be zero:

$$
\begin{aligned}
H= & \chi\left\{\hat{a}^{\dagger 2} \hat{a}^{2}\right\}_{s}+g_{1}\left(\hat{a} \mathrm{e}^{\mathrm{i} \Omega t}+\hat{a}^{\dagger} \mathrm{e}^{-\mathrm{i} \Omega t}\right) \\
& +g_{2}\left(\hat{a}^{2} \mathrm{e}^{\mathrm{i} \Omega t}+\hat{a}^{\dagger 2} \mathrm{e}^{-\mathrm{i} \Omega t}\right)
\end{aligned}
$$

Clearly, Eq. (4) may equally well be expressed in normal ordering using $\left\{\hat{a}^{\dagger 2} \hat{a}^{2}\right\}_{s}=a^{\dagger 2} \hat{a}^{2}+$ $2 \hbar \hat{a}^{\dagger} a+\hbar^{2} / 2$. The time-evolution operator $U(T)$ has been calculated by numerically solving the equation of motion

$$
\mathrm{i} \hbar \partial_{t} U(t)=H(t) U(t)
$$

(different algorithms are available; here we used a straightforward Runge-Kutta integration of the coupled equations for the expansion coefficients in the oscillator basis $\left.a^{\dagger} a|n\rangle=\hbar n|n\rangle\right)$. The propagated matrix $U_{n m}(T)=\langle n|U(T)| m\rangle$ has been diagonalized to yield the Floquet eigenstates at time zero

$$
U(T)\left|\psi_{\alpha}(0)\right\rangle=\mathrm{e}^{-\mathrm{i} \epsilon_{\alpha} T / \hbar}\left|\psi_{\alpha}(0)\right\rangle,
$$

with components $\psi_{n}^{\alpha}=\left\langle n \mid \psi_{\alpha}(0)\right\rangle$ as well as the eigenvalues $\epsilon_{\alpha}$.

For the semiclassical construction of the propagator, we need the classical Hamilton function $H(x, p)$, which - due to our choice of (4) - is independent of $\hbar$, i.e.:

$$
\begin{aligned}
H(p, q)= & \frac{\chi}{4}\left(p^{2}+q^{2}\right)^{2}+\sqrt{2} g_{1}(q \cos (\Omega t)-p \sin (\Omega t)) \\
& +g_{2}\left(\left(q^{2}-p^{2}\right) \cos (\Omega t)-2 q p \sin (\Omega t)\right)
\end{aligned}
$$

Following the semiclassical frozen Gaussian FGA [10], we approximate the true propagator by

$$
U^{\mathrm{scl}}(t)=\int \frac{d p_{i} d q_{i}}{2 \pi \hbar} A\left(p_{i}, q_{i}, t\right) \mathrm{e}^{\mathrm{i} S\left(p_{i}, q_{i}, t\right) / \hbar}\left|p_{f}, q_{f}\right\rangle\left\langle p_{i}, q_{i}\right|
$$


where $|p, q\rangle=\mathrm{e}^{i(p \hat{q}-q \hat{p}) / \hbar}|0\rangle$ are coherent states with $\hat{a}|0\rangle=0$ (see (12) for a representation in harmonic oscillator eigenfunctions). In other words, $\left|q_{i}, p_{i}\right\rangle$ is a minimum uncertainty Gaussian wave packet centered at the initial phase space point $\left(p_{i}, q_{i}\right)$, and $\left|p_{f}, q_{f}\right\rangle$ is centered at the time-evolved position of the classical trajectory at $\left(p_{f}, q_{f}\right)=(p(t), q(t))$. The phase is essentially determined by an action integral

$$
S\left(p_{i}, q_{i}, t\right)=-\frac{1}{2}\left(p_{f} q_{f}-p_{i} q_{i}\right)+\int_{0}^{t}[p \dot{q}-H(p, q, \tau)] d t
$$

and the amplitude is given by

$$
A^{2}\left(q_{i}, p_{i}, t\right)=\frac{1}{2}\left(\frac{\partial p_{f}}{\partial p_{i}}+\frac{\partial q_{f}}{\partial q_{i}}+\mathrm{i} \frac{\partial p_{f}}{\partial q_{i}}-\mathrm{i} \frac{\partial q_{f}}{\partial p_{i}}\right) .
$$

In an equivalent formulation, the terms $-p_{i} q_{i} / 2$ and $p_{f} q_{f} / 2$ can be included in the definition of the coherent states. It seems worthwhile to emphasize that, in contrast to related semiclassical methods, only the amplitude and the phase of the Gaussians are varied along with the classical propagation, but not their size and shape.

The fact that the action $S$ given in Eq. (9) holds for time dependent Hamiltonians as well is immediately seen by using the $\left(t, t^{\prime}\right)$ formalism to solve the Schrödinger equation [25]. Since following the $\left(t, t^{\prime}\right)$ (extended phase space) formalism, $t^{\prime}$ appears as an additional coordinate and $H$ is replaced by the Floquet Hamiltonian [26] so that the classical term $p_{t^{\prime}} \dot{t}^{\prime}=p_{t}^{\prime}$ is canceled out when $-H$ is replaced by $-\left(H+p_{t^{\prime}}\right)=-H_{F}$.

The action (9) as well as the partial derivatives in the prefactor (10) have been calculated by propagating the monodromy matrix simultaneously with the trajectories [27]. After calculating all the classical elements, the approximative propagator has been evaluated by an integration,

$$
U_{n m}^{\mathrm{scl}}(T)=\int \frac{d p_{i} d q_{i}}{2 \pi \hbar} A(T) \mathrm{e}^{\mathrm{i} S(T) / \hbar}\left\langle n \mid p_{f}, q_{f}\right\rangle\left\langle p_{i}, q_{i} \mid m\right\rangle
$$

with

$$
\langle n \mid p, q\rangle=\mathrm{e}^{-\left(p^{2}+q^{2}\right) / 4 \hbar} \frac{1}{\sqrt{n !}}\left(\frac{q+\mathrm{i} p}{\sqrt{2 \hbar}}\right)^{n},
$$


to yield the same form as in the exact numerical quantum calculation (5). Both, the amplitude $A\left(p_{i}, q_{i}, T\right)$ and the action $S\left(p_{i}, q_{i}, T\right)$, depend smoothly on the initial conditions $\left(p_{i}, q_{i}\right)$.

Figure 1 shows a classical stroboscopic Poincaré section for the driven anharmonic oscillator (7). The parameters are $\chi=0.1, g_{1}=0.005, g_{2}=0.01$, and $\Omega=0.5$. For these parameters, the dynamics is clearly organized: In the outer region, where the driving frequency is small compared to the anharmonic motion in the time independent potential, and in the inner region, where the reverse relation holds, the dynamics is almost regular. The 1:1 resonance developed into an extended chaotic 'sea' and a stable resonance island centered at $(p, q) \approx(0,-2.2)$. In addition, one observes two smaller islands of the 1:2 resonance at $(p, q) \approx( \pm 1.6,0.15)$.

These classical phase space structures can also be detected in the quantum quasienergy states (we use the same parameters as in the classical case and $\hbar=0.02$ ). The quasienergy states are numbered by $\alpha=0,1, \ldots$ with increasing values of the expectation value $\left\langle H_{0}\right\rangle_{\alpha}=$ $\left\langle\psi_{\alpha}(0)\left|H_{0}\right| \psi_{\alpha}(0)\right\rangle$ of the field-free Hamiltonian $H_{0}$.

A quantitative description of the localization properties in phase space is provided by the Husimi distributions [28]

$$
\varrho_{\alpha}(p, q)=\left|\left\langle p, q \mid \psi_{\alpha}(0)\right\rangle\right|^{2}
$$

The entropy

$$
S_{\alpha}=-\int \frac{d p d q}{2 \pi \hbar} \varrho_{\alpha}(p, q) \ln \left[2 \pi \hbar \varrho_{\alpha}(p, q)\right]
$$

of the Husimi distribution is a measure of the phase space area occupied by state $\alpha$. An energy-entropy diagram as shown in Fig. 2 can be used to separate the states into different classes according to their phase space localization $[3,4]$. Here we distinguish six classes labeled by $A, \ldots, F$ in Fig. 2. States of class $A$ localize on the central island and class $B$ consist of almost degenerate pairs localizing on the two 1:2 resonance islands. About 50 states in class $C$ can be considered as 'chaotic', i.e. their Husimi distribution shows seemingly 
erratic oscillation over the classically chaotic region; the nearest neighbour spacings of the quasienergies are Wigner distributed [4], and the entropy (14) shows strong fluctuations $\sqrt{\hbar}[4]$ with an average value of $\bar{S}^{\mathrm{qm}} \approx 3.73 \pm 0.4$. In agreement with previous findings [4], this quantum value is considerably smaller than the classical estimate $\bar{S}^{\text {cl }}=\ln \left(A_{\text {chaotic }} /(2 \pi \hbar)\right) \approx$ 4.40 , where $A_{\text {chaotic }} \approx 10.24$ is the area of the classically chaotic region. The difference $\bar{S}^{\mathrm{cl}}-\bar{S}^{\mathrm{qm}}=0.67$ should approach the value of $c_{2}=1-c=0.423 \ldots(c=$ Euler's number $)$ for small $\hbar$ [4]. The class $D$ states in Fig. 2 are supported by the $1: 1$ resonance at $(p, q) \approx$ $(0,-2.2)$ in Fig. 1, where the state $\alpha=108$ with the lowest energy and entropy in this class localizes at the center of the resonance island and the higher entropy and energy states approach finally the outer separatrix, where the cloud of class $C$ states meets the separatrix states $E$. Class $F$ consists of the outer regular states. This classification can also be verified by inspection of the individual Husimi distributions in phase space.

The main result of the present letter is, however, the possibility to reproduce the entire quantum quasienergy spectrum semiclassically. Figure 3 shows a comparison of quantum and semiclassical quasiangles $\theta_{\alpha}=\epsilon_{\alpha} T / \hbar$, which shows a surprisingly good agreement for both regular and chaotic states. Note that the quasienergies $\epsilon_{\alpha}$ are given modulo $\hbar \omega$, i.e. $\theta_{\alpha}$ modulo $2 \pi$. For all states shown in the figure, the difference is less than $10^{-2}$ (in the figure, the real part of the semiclassical quasiangle is shown; the (small) imaginary part is due to the fact, that the FGA is unitary only within the stationary phase approximation [9]). For such a comparison as shown in Fig. 3, the semiclassical states corresponding to the quantum ones first must be identified. This is done by selecting the quantum state with maximum overlap with the semiclassical one, which turns out to be a quite secure recipe, because this overlap is very close to unity or zero (see below). It should be pointed out, that the semiclassical FGA method also seems to reproduce the tunneling splittings of the pairs of states of class $B$, namely $\alpha=(50,51),(49,53)$, and $(41,58)$ localizing on the $1: 2$ resonance islands. For these states, the quasiangles are degenerate modulo $\pi$ [6] and the splittings are $4.03 \cdot 10^{-7}, 3.4 \cdot 10^{-4}$ and $1.6 \cdot 10^{-3}$, respectively, in satisfactory agreement with the semiclassical splittings $4.01 \cdot 10^{-7}, 2.1 \cdot 10^{-4}$ and $1.7 \cdot 10^{-3}$. 
The semiclassical eigenfunctions, i.e. the eigenstates of the semiclassical propagator, are of the same accuracy as the eigenvalues. As an illustration, Fig. 4 shows a comparison of the quantum and semiclassical Husimi distributions for the state $\alpha=87$, which is the state with the largest entropy localizing on the chaotic phase space region in Fig. 1. The two distributions are (almost) identical.

Let us furthermore point out that the proposed method is free from the well-known drawbacks of other semiclassical methods. It is, however, clearly not fully semiclassical. The matrix elements (11) are computed numerically and not by, e.g., stationary phase and the eigenvalues and eigenvectors of the Floquet operator are obtained exactly as in quantum mechanics. The basic input for the semiclassical method, however, the Floquet propagator, is entirely taken from classical dynamics.

In conclusion, we have demonstrated that the semiclassical initial value representation can be used to compute the entire spectrum for both regular and chaotic stated from classical dynamics. However, one should note that in the present 1D case the computational 'costs' of both computations are of the same order of magnitude. This is very different in higher dimensions, where the semiclassical method can be expected to be much faster (see, e.g. the recent work on photodissociation of ozone [29] using the semiclassical initial value representation.) In addition, the semiclassical method provides illuminating insight into the quantum dynamics, i.e. the phase space localization properties of quantum states on (classically) regular and chaotic regions.

This work was done while one of the authors (N.M.) was staying at the University of Kaiserslautern as the 1997 Humboldt awardee. The research has been supported by the Deutsche Forschungsgemeinschaft (SPP 'Zeitabhängige Phänomene und Methoden in Quantensystemen der Physik und Chemie'). 


\section{REFERENCES}

[1] N. Ben-Tal, N. Moiseyev, and H. J. Korsch, Phys. Rev. A 46, 1669 (1992); N. Ben-Tal, N. Moiseyev, S. Fishman, F. Bensch, and H. J. Korsch, Phys. Rev. E 47, 1646 (1993).

[2] B. Mirbach and H. J. Korsch, Phys. Rev. Lett. 75, 362 (1995).

[3] N. Moiseyev, H. J. Korsch, and B. Mirbach, Z. Phys. D 29, 125 (1994).

[4] T. Gorin, H. J. Korsch, and B. Mirbach, Chem. Phys. 217, 145 (1997).

[5] H. P. Breuer and M. Holthaus, Ann. Phys. (N.Y.) 211, 249 (1991).

[6] F. Bensch, H. J. Korsch, B. Mirbach, and N. Ben-Tal, J. Phys. A 25, 6761 (1992); B. Mirbach and H. J. Korsch, J. Phys. A 27, 6579 (1994); B. Mirbach H. J. Korsch and B. Schellhaaß, Phys. Rev. A 30, 1659 (1997).

[7] W. H. Miller, J. Chem. Phys. 53, 3578 (1970).

[8] E. J. Heller, J. Chem. Phys. 75, 2923 (1981).

[9] M. F. Hermann, J. Chem. Phys. 85, 2069 (1984).

[10] M. F. Hermann and E. Kluk, Chem. Phys. 91, 27 (1984); E. Kluk, M. F. Hermann, and H. L. Davis, J. Chem. Phys. 84, 326 (1986).

[11] R. Hernandez and W. H. Miller, Chem. Phys. Lett. 214, 129 (1994); S. Keshavamurthy and W. H. Miller, Chem. Phys. Lett. 218, 189 (1994); B. W. Spath and W. H. Miller, Chem. Phys. Lett. 262, 486 (1996); J. Chem. Phys. 104, 95 (1996).

[12] F. Grossmann, Chem. Phys. Lett. 262, 470 (1996).

[13] S. Garashchuk and D. Tannor, Chem. Phys. Lett. 262, 477 (1996).

[14] A. R. Walton and D. E. Manolopoulos, Chem. Phys. Lett. 244, 448 (1995).

[15] K. G. Kay, J. Chem. Phys. 100, 4377 (1994); J. Chem. Phys. 100), 4432 (1994). 
[16] M. A. Sepúlveda and E. J. Heller, J. Chem. Phys. 101, 8004; 8016 (1994).

[17] A. R. Walton and D. E. Manolopoulos, Molec. Phys. 87, 961 (1996); M. L. Brewer, J. S. Hulme, and D. E. Manololoulos, J. Chem. Phys. 106, 4832 (1997).

[18] M. A. Sepúlveda and F. Grossmann, Adv. Chem Phys. XCVI, 191 (1996).

[19] A. K. Pattanayak and W. C. Schieve, Phys. Rev. E 56, 278 (1997).

[20] F. Grossmann, V. A. Mandelshtam and H. S. Taylor and J. S. Briggs, Chem. Phys. lett. 279, 355 (1997).

[21] M. C. Gutzwiller, Chaos in Classical and Quantum Mechanics (Springer, New York, 1990).

[22] P. Gerwinski, F. Haake, H. Wiedemann, M. Kuś, and K. Życzkowski, Phys. Rev. Lett. 74, 1562 (1995).

[23] S.-J.. Chang and G. Perez, Phys. Rev. A 42, 5898 (1990).

[24] L. E. Reichl, The Transition to Chaos (Springer, New York, 1992).

[25] U. Peskin and N. Moiseyev, J. Chem. Phys. 99, 4590 (1993).

[26] N. Moiseyev and F. Weinhold, Phys. Rev. Lett 78, 2100 (1997).

[27] H. D. Meyer, Phys. Rev. A 84, 3147 (1986).

[28] K. Takahashi, Progr. Theor. Phys. Suppl. 98, 109 (1989).

[29] V. S. Batista and W. H. Miller, J. Chem. Phys. 108, 498 (1998). 


\section{FIGURES}

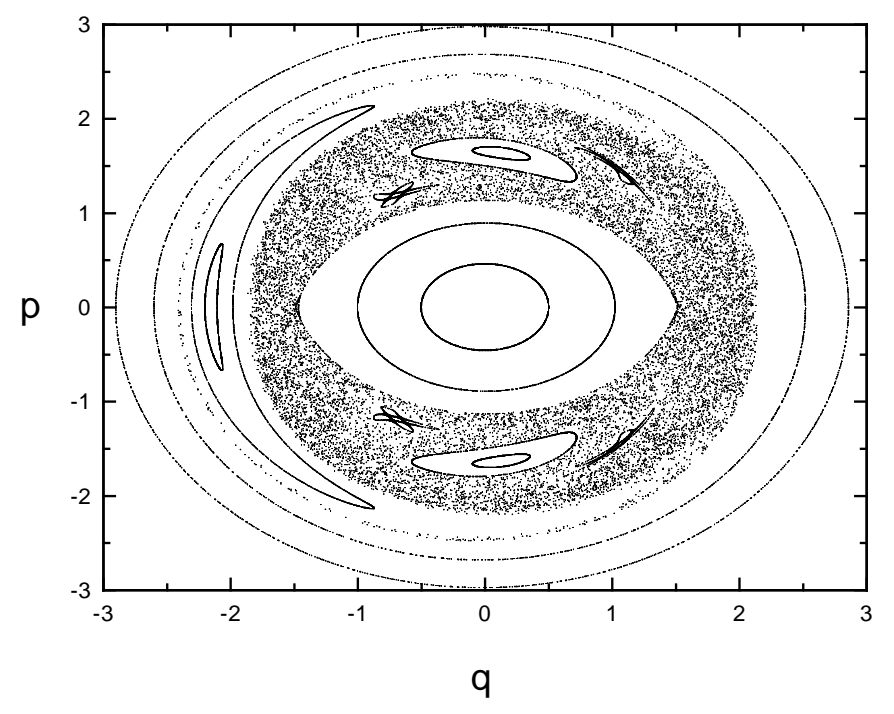

FIG. 1. Classical stroboscopic Poincaré section for the driven anharmonic oscillator (7).

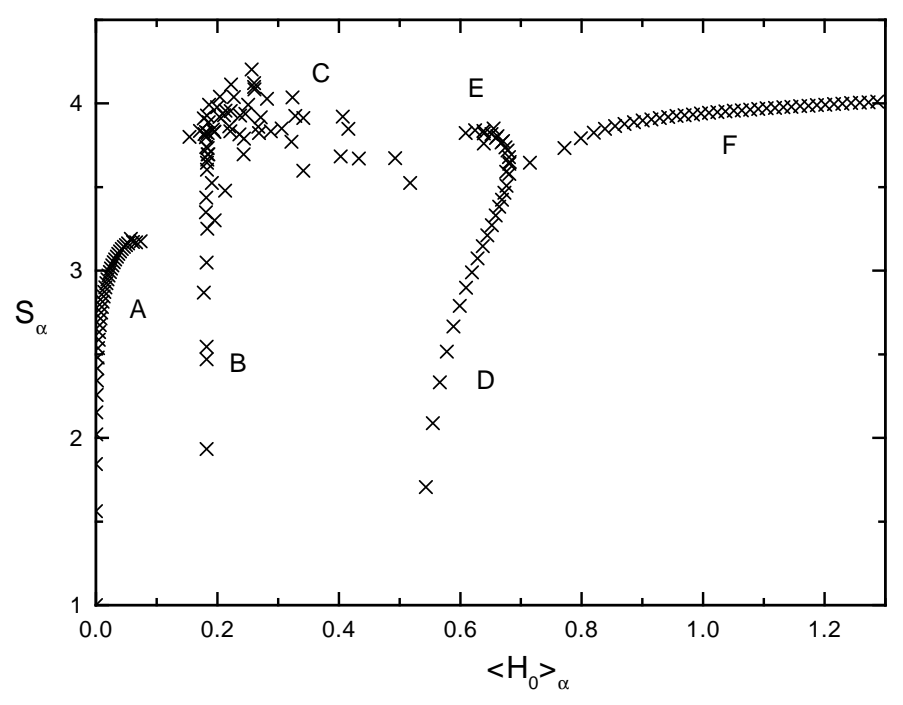

FIG. 2. Husimi entropy of the quasienergy states versus the expectation value of the field-free Hamiltonian (same parameters as in Fig. $1 ; \hbar=0.02$ ). 


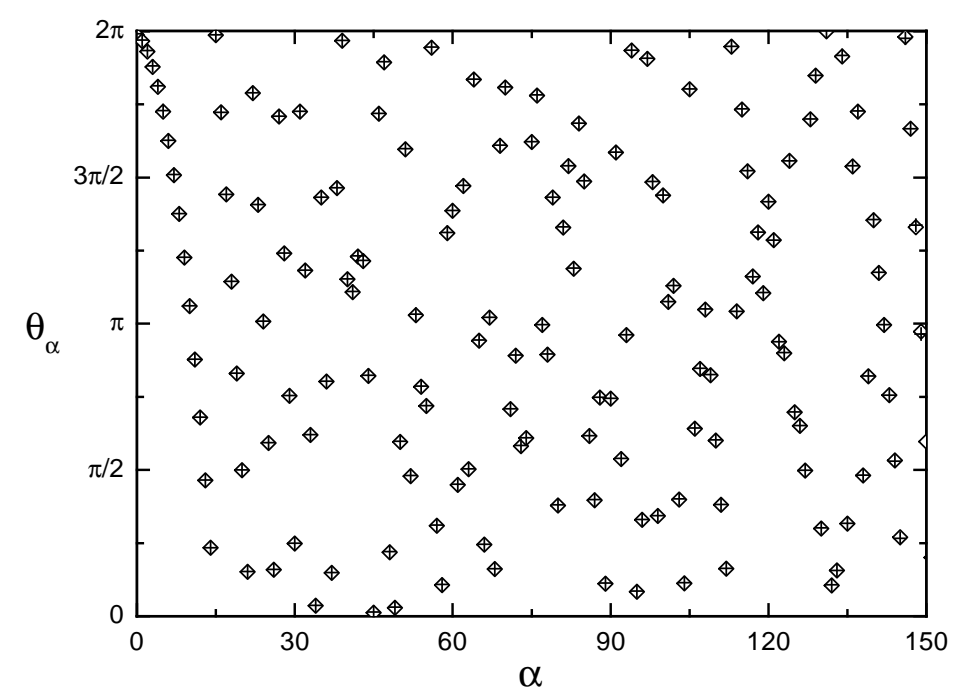

FIG. 3. Comparison of quantum $(\diamond)$ and semiclassical quasi angle $\theta_{\alpha}=\epsilon_{\alpha} T / \hbar(+)$. The quasienergies $\epsilon_{\alpha}$ are given modulo $\hbar \omega$, i.e. $\theta_{\alpha}$ modulo $2 \pi$.

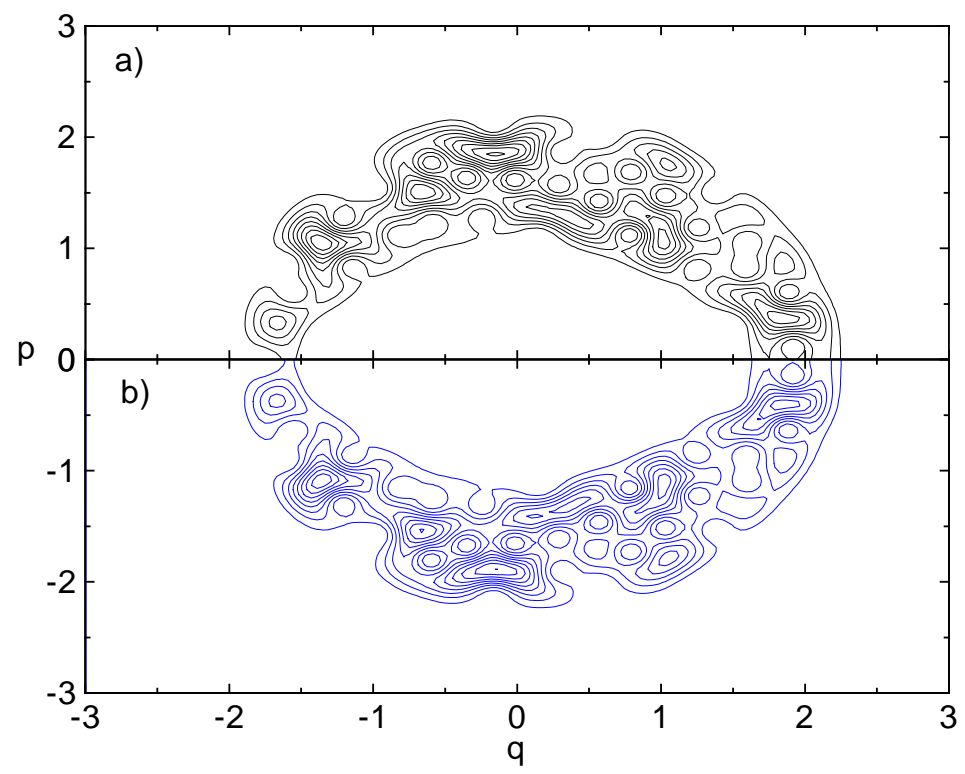

FIG. 4. Quantum (a) and semiclassical (b) Husimi distributions for the state $\alpha=87$. This state has the largest entropy and is localized on the chaotic phase space region in Fig. 1. Because of symmetry, only one half of the distributions are displayed. 